Shlomi Laufer, Ph.D.

Anne-Lise D. D'Angelo, M.D.

Carla M. Pugh, M.D., Ph.D.

University of Wisconsin School of Medicine and Public Health Madison, WI

and Others

A complete list of authors is provided with the full text of this letter at NEJM.org.

Supported by grants from the National Institutes of Health (R01EB011524, to Dr. Pugh; and 1F32EB017084-01, to Dr. D'Angelo).

Disclosure forms provided by the authors are available with the full text of this letter at NEJM.org.
1. Epstein RM. Assessment in medical education. $\mathrm{N}$ Engl J Med 2007;356:387-96.

2. Norcini JJ, Lipner RS, Grosso LJ. Assessment in the context of licensure and certification. Teach Learn Med 2013;25:Suppl 1: S62-S67.

3. Reznick RK, MacRae H. Teaching surgical skills - changes in the wind. N Engl J Med 2006;355:2664-9.

4. Kogan JR, Holmboe ES, Hauer KE. Tools for direct observation and assessment of clinical skills of medical trainees: a systematic review. JAMA 2009;302:1316-26.

5. Saslow D, Hannan J, Osuch J, et al. Clinical breast examination: practical recommendations for optimizing performance and reporting. CA Cancer J Clin 2004;54:327-44.

DOI: 10.1056/NEJMc1414210

\title{
Viremic Relapse after HIV-1 Remission in a Perinatally Infected Child
}

TO THE EDITOR: We previously reported ${ }^{1}$ the remission of human immunodeficiency virus type 1 (HIV-1) in a perinatally infected child - the "Mississippi Child." After receiving antiretroviral therapy (ART) between 30 hours and 18 months of age, this child had persistently undetectable plasma HIV-1 viremia for 12 months in the absence of HIV-1-specific immune responses. At the time of the initial case report, it was uncertain whether HIV-1 reservoirs were established that could lead to rebound viremia.

After the first report, the child had persistently undetectable plasma HIV-1 RNA levels, as assessed by means of standard clinical assays, with normal CD4+ and CD8+ T-cell counts. Plasma viremia also remained undetectable through 21.9 months after the discontinuation of ART on ultrasensitive single-copy viral-load assays ( $<0.4$ and $<0.5$ copies per milliliter).

During routine clinical follow-up at 46.4 months of age (27.6 months after the discontinuation of ART), the plasma viral load rebounded to 16,750 copies per milliliter; this was confirmed with repeat testing (Fig. 1A). The child did not have symptoms or signs of an acute retroviral syndrome or incident illnesses (including EpsteinBarr virus infection or cytomegalovirus infection) and had not received any vaccines. Epidemiologic risk factors for reinfection with HIV-1 such as through breast milk, premasticated food, or sexual abuse were not identified.
Within 72 hours after the reinitiation of ART, the plasma viral load dropped to 2658 copies per milliliter (Fig. 1A), circulating CD4+ T-cell levels increased to $43 \%$, and the ratio of CD4+ T cells to $\mathrm{CD} 8+\mathrm{T}$ cells normalized.

During the period of virologic remission, HIV-1 DNA was intermittently detected in circulating unfractionated peripheral-blood mononuclear cells (PBMCs) and cells enriched for resting and activated CD4+ T cells. HLA typing confirmed the patient identity of the cellular DNA used for HIV-1 DNA measurements. The median number of copies of HIV-1 DNA in unfractionated PBMCs and resting and activated CD4+ T cells before rebound viremia was 2.7 per million cells (interquartile range, 2.2 to 3.4 ), 3.5 per million cells (interquartile range, 2.1 to 4.4), and 3.3 per million cells (interquartile range, 1.8 to 5.5), respectively. However, replicationcompetent viral reservoirs remained undetectable throughout follow-up in a cumulative 64 million cultured resting CD4+ T cells.

HIV-1-specific antibody and cell-mediated immune responses were not detected through 21.9 months after the discontinuation of ART but were detected at viremic rebound by means of enzyme-linked immunosorbent assay. The findings were confirmed by Western blot testing (1:10 dilution), which revealed reactivity to HIV-1 Env (gp160) and Gag (p24); 72 hours later, Western blot testing revealed reactivity to HIV-1 Env 
Figure 1. Plasma HIV-1 Load and Phylogenetic Analyses. Panel A shows human immunodeficiency virus type 1 (HIV-l) RNA levels during antiretroviral treatment (ART), while the child was not receiving ART, and after the reinitiation of ART. Solid circles indicate detectable HIV-I RNA, and open circles undetectable HIV-1 RNA; the shaded area indicates the limit of detection of the plasma viral-load assay. 3TC denotes lamivudine, AZT zidovudine, EFV efavirenz, LPV/r ritonavir-boosted lopinavir, and NVP nevirapine. Panel B shows phylogenetic analyses of maternal plasma viral sequences amplified from a maternal plasma sample collected 24 months after delivery of the infant (MOl) and during viral rebound in the infant (BOl) (shaded area). The neighborjoining tree depicts full-length gpl60 env sequences. Four previously described ${ }^{2}$ HIV-1 clade B-infected mother-infant pairs were included in the analysis for comparison of interpatient diversity and relatedness between mother and infant.

(gp160, gp120, and gp41), Gag (p55, p24, and p17), and Pol (p66). The lack of detectable responses to p31 integrase was compatible with rebound viral replication of at least 1 month but less than 2 months. ${ }^{3}$

Before viremic rebound, sequencing attempts were unsuccessful. Single-genome amplification and sequencing of full-length HIV-1 Env directly from rebounding plasma virus revealed $98.6 \%$ sequence identity to maternal plasma viral sequences collected 24 months after delivery (Fig. 1B). Diversity was $0.1 \%$, consistent with values seen in transmitted founder viruses in motherto-child HIV-1 transmission ${ }^{4}$ and a lack of ongoing virus replication during the period of HIV-1 remission. Phenotypic and genotypic analysis of the HIV-1 Env sequence from rebounding virus showed R5 tropism.

In conclusion, the return of HIV-1 viremia after a substantial period of viral quiescence is consistent with the model of HIV-1 latency in which long-lived resting memory CD4+ T cells were generated during routine immunologic memory formation, though other reservoirs are also possible. To the best of our knowledge, this child received ART between 30 hours and 18 months of age. Whether earlier initiation or a longer duration of ART, alone or combined with immunotherapeutic strategies, would have affected the duration of remission is unknown. The findings in this case and others ${ }^{5}$ provide

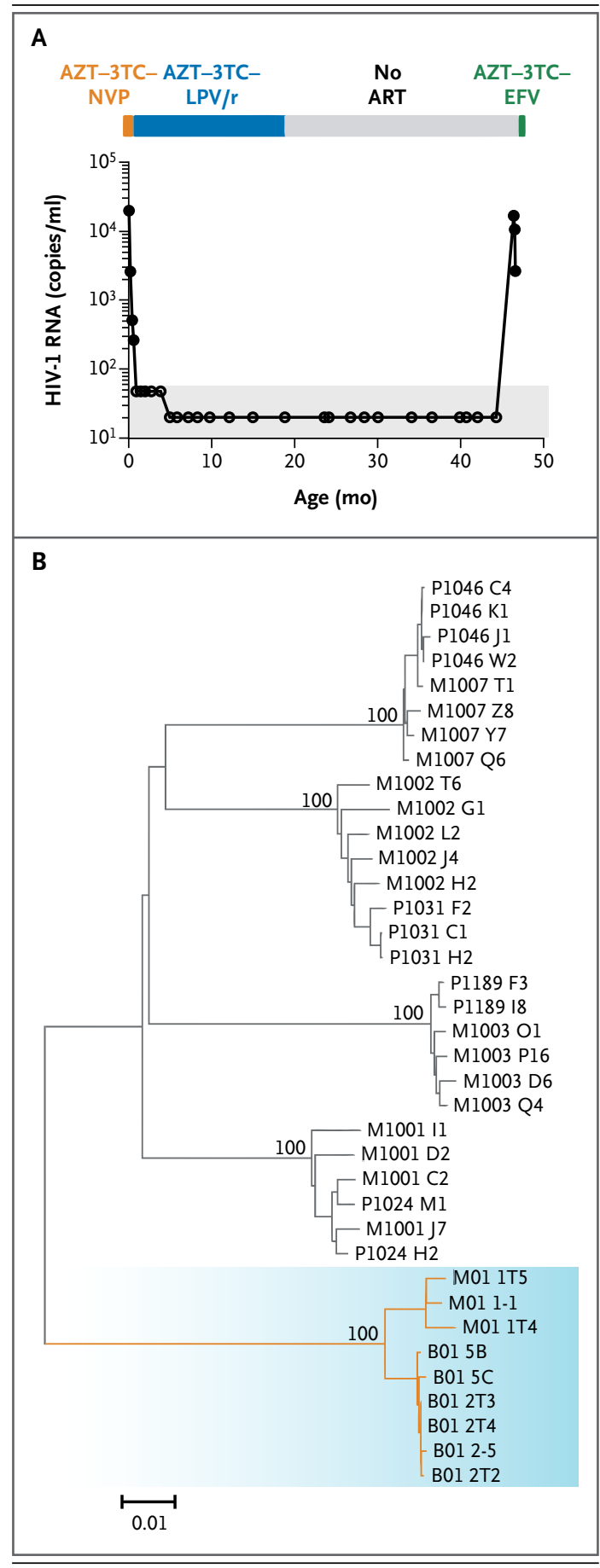

insight on how very early treatment may restrict but not eradicate HIV-1 reservoirs. Additional studies are under way to test this hypothesis and to determine whether ART alone will enable longer-term HIV-1 remission. Until these studies 
are completed, initiation of ART as early as possible in infants and continuation without interruption seem prudent.

Katherine Luzuriaga, M.D.

University of Massachusetts Medical School Worcester, MA

Hannah Gay, M.D.

University of Mississippi Medical Center Jackson, MS

Carrie Ziemniak, M.S.

Johns Hopkins University School of Medicine Baltimore, MD

Keri B. Sanborn, Ph.D.

Mohan Somasundaran, Ph.D.

University of Massachusetts Medical School

Worcester, MA

Kaitlin Rainwater-Lovett, Ph.D.

Johns Hopkins University School of Medicine Baltimore, MD

John W. Mellors, M.D.

University of Pittsburgh

Pittsburgh, PA

Daniel Rosenbloom, Ph.D.

Columbia University

New York, NY

Deborah Persaud, M.D.

Johns Hopkins University School of Medicine Baltimore, MD

dpers@jhmi.edu

The content of this letter does not necessarily reflect the views or policies of the Department of Health and Human Services, nor does mention of trade names, commercial products, or organizations imply endorsement by the U.S. government.

Supported by grants from the National Institutes of Health (RO1 HD080474, to Drs. Persaud and Luzuriaga; AI1097265, to Dr. Luzuriaga; and F32 AI108414, to Dr. Sanborn), the Johns Hopkins University Center for AIDS Research (P30AI094189, to Dr. Persaud), the International Maternal Pediatric Adolescent AIDS Clinical Trials Network (UM1 AI106716, to Dr. Persaud), and the University of Massachusetts Center for Clinical and Translational Science (UL1TR000161, to Dr. Luzuriaga). Dr. Mellors is supported by a grant (UM1AI106701) from the AIDS Clinical Trials Group to the Pittsburgh Virology Specialty Laboratory and by a grant (contract 12XS547) from Leidos Biomedical Research through the National Cancer Institute.

Disclosure forms provided by the authors are available with the full text of this letter at NEJM.org.

1. Persaud D, Gay H, Ziemniak C, et al. Absence of detectable HIV-1 viremia after treatment cessation in an infant. N Engl J Med 2013;369:1828-35.

2. Kishko M, Somasundaran M, Brewster F, et al. Genotypic and functional properties of early infant HIV-1 envelopes. Retrovirology 2011;8:67.

3. Tomaras GD, Haynes BF. HIV-1-specific antibody responses during acute and chronic HIV-1 infection. Curr Opin HIV AIDS 2009;4:373-9.
4. Wolinsky SM, Wike CM, Korber BT, et al. Selective transmission of human immunodeficiency virus type-1 variants from mothers to infants. Science 1992;255:1134-7.

5. Rainwater-Lovett K, Luzuriaga K, Persaud D. Very early combination antiretroviral therapy in infants: prospects for cure. Curr Opin HIV AIDS 2015;10:4-11.

DOI: 10.1056/NEJMc1413931

Correspondence Copyright (c) 2015 Massachusetts Medical Society.

\section{INSTRUCTIONS FOR LETTERS TO THE EDITOR}

Letters to the Editor are considered for publication, subject to editing and abridgment, provided they do not contain material that has been submitted or published elsewhere. Please note the following:

- Letters in reference to a Journal article must not exceed 175 words (excluding references) and must be received within 3 weeks after publication of the article.

- Letters not related to a Journal article must not exceed 400 words.

- A letter can have no more than five references and one figure or table.

- A letter can be signed by no more than three authors.

- Financial associations or other possible conflicts of interest must be disclosed. Disclosures will be published with the letters. (For authors of Journal articles who are responding to letters, we will only publish new relevant relationships that have developed since publication of the article.)

- Include your full mailing address, telephone number, fax number, and e-mail address with your letter.

- All letters must be submitted at authors.NEJM.org.

Letters that do not adhere to these instructions will not be considered. We will notify you when we have made a decision about possible publication. Letters regarding a recent Journal article may be shared with the authors of that article. We are unable to provide prepublication proofs. Submission of a letter constitutes permission for the Massachusetts Medical Society, its licensees, and its assignees to use it in the Journal's various print and electronic publications and in collections, revisions, and any other form or medium.

NOTICES

Notices submitted for publication should contain a mailing address and telephone number of a contact person or department. We regret that we are unable to publish all notices received. Notices also appear on the Journal's website (NEJM.org/medical-conference). The listings can be viewed in their entirety or filtered by specialty, location, or month.

\section{TH AMERICAN COUGH CONFERENCE}

The conference will be held in Washington, D.C., June 5 and 6. Contact Judi Miller, Strategic Pharmaceutical Advisors, 1750 Tysons Blvd., Suite 1500, McLean, VA 22101; or call (202) 9652288; or fax (202) 747-3415; or e-mail JMiller@SRxA.com; or see http://www.americancoughconference.com. 